\title{
Excitation of stellar p-modes by turbulent convection
}

\section{Theoretical formulation}

\author{
R. Samadi ${ }^{1,2}$ and M.-J. Goupil ${ }^{3}$ \\ 1 Observatoire de Paris, DESPA, CNRS UMR 8632, 92195 Meudon, France \\ 2 Astronomy Unit, Queen Mary, University of London, London E14NS, UK \\ 3 Observatoire de Paris, DASGAL, CNRS UMR 8633, 92195 Meudon, France
}

Received 17 October 2000 / Accepted 19 January 2001

\begin{abstract}
Stochatic excitation of stellar oscillations by turbulent convection is investigated and an expression for the power injected into the oscillations by the turbulent convection of the outer layers is derived which takes into account excitation through turbulent Reynolds stresses and turbulent entropy fluctuations. This formulation generalizes results from previous works and is built so as to enable investigations of various possible spatial and temporal spectra of stellar turbulent convection. For the Reynolds stress contribution and assuming the Kolmogorov spectrum we obtain a similar formulation to those derived by previous authors. The entropy contribution to excitation is found to originate from the advection of the Eulerian entropy fluctuations by the turbulent velocity field. Numerical computations in the solar case in a companion paper indicate that the entropy source term is dominant over the Reynold stress contribution to mode excitation, except at high frequencies.
\end{abstract}

Key words. convection - turbulence - stars: oscillations - Sun: oscillations

\section{Introduction}

Oscillation amplitudes and linewidths provide information on excitation and damping processes of stellar oscillation modes. In the solar case, the observed oscillation modes are believed to be damped as a result of a competition between several non-adiabatic and turbulent processes (Osaki 1990; Houdek et al. 1999). Excitation of solar oscillation modes is attributed to turbulent convection at the surface of the Sun. The first theoretical investigation of solar oscillation excitation by turbulent convection was by Goldreich \& Keeley (1977, hereafter GK). These authors identified the turbulent term of Reynolds stress as the main source term of stochastic excitation of solar acoustic modes in the wave equation. GK derived an approximate estimation for the acoustic power injected into the oscillations by turbulent convection which arises from an equipartition of energy between the turbulent elements and the oscillations. The result, however, underestimated the power by a factor $\sim 10^{3}$ compared to the solar observations (Osaki 1990). Amplitude estimations for solar-like oscillating stars have subsequently been computed by Christensen-Dalsgaard \& Frandsen (1983) based on this simple picture of equipartition of energy between turbulence and oscillation. GK considered the adiabatic

Send offprint requests to: R. Samadi, e-mail: Reza.Samadi@obspm.fr assumption for both the oscillations and the turbulence. A decade later, entropy turbulent fluctuation has been proposed as a possible additional excitation source (Stein \& Nordlund 1991; Balmforth 1992; Goldreich et al. 1994).

The stochastic mechanism may be understood as follows: turbulent motions of the stellar material and turbulent fluctuations of thermodynamicals quantities, which occur in the convection zone, generate acoustic waves (Lighthill 1952; Stein 1967). The corresponding acoustic power then excites resonant modes of the stellar cavity (oscillations). This excitation of the oscillations results from a forcing by incoherent (turbulent) source terms due to the turbulent Reynolds stress and turbulent entropy fluctuations.

An alternative formulation for the power and amplitude oscillation is proposed by Balmforth (1992, hereafter B92) and is used by Houdek et al. (1999) to compute amplitude of oscillation for various solar-type stars. There is some disagreement between the conclusions of both formulations concerning the entropy contribution: it is found dominant in theoretical investigation (Goldreich et al. 1994, hereafter GMK) and as a result of numerical 3D simulations (Stein \& Nordlund 1991), but negligible in some other cases (Balmforth 1992).

Both formulations are built following the method developed by GK and are based on a simplified description of the turbulent medium. The spectrum of turbulent 
energy in stellar conditions is, however, not well known. The best-known spectrum is, of course, the solar spectrum (Espagnet et al. 1993; Nesis et al. 1993) and observations tell us that a Kolmogorov spectrum does not represent the complete turbulent solar spectrum (Nesis et al. 1993).

The purpose of the present work therefore is to investigate the effect of several possible models of turbulence on the excitation of solar-like oscillation modes and to establish their signature in power spectra. To do so, a formulation must first be established which includes both (kinetic and entropic) contributions in a consistent and general way. The main goal is to allow the use of any form of the turbulent kinetic energy spectrum, of turbulent entropy fluctuations and the eddy temporal spectrum. Two free parameters are introduced to take into account uncertainties in the definitions of the coherence time of the turbulent eddies and in the magnitude of the wavenumber at which the inertial regime starts.

Once the general formulation is established, we study the specific case of a Gaussian time and Kolmogorov energy spectra, which enables us to compare our findings with results of previous works. In a companion paper, the present formulation is applied to the solar case and several turbulent models found in the literature are discussed. Both free parameters can be calibrated with solar observations and used to compute oscillation amplitudes for other potential solar-like oscillating stars (Samadi et al. $2001 b, 2001 c$ ) in view of forthcoming space seismological experiments: COROT (Baglin \& The Corot Team 1998), MONS (Kjeldsen \& Bedding 1998), MOST (Matthews 1998), EDDINGTON (Favata et al. 2000).

Section 1 recalls how the stochastic excitation mechanism of stellar oscillations is modeled by source terms in the wave equation. We assume the modes are damped and find that they are excited through the turbulent Reynolds stresses (Goldreich \& Keeley 1977) and the turbulent entropy fluctuations arising from the turbulent nature of the stellar convection region (Stein \& Nordlund 1991; Balmforth 1992; Goldreich et al. 1994). We adopt the GK procedure and assume that the oscillation modes are decoupled from the turbulent medium. This description gives rise to an inhomogeneous wave equation for the oscillation modes. In this framework, the acoustic turbulent source acts as a forcing term for the oscillation normal modes. We consider adiabatic radial oscillations in the Cowling (1941) approximation. A homogeneous, isotropic, stationary turbulence is assumed. A formulation for the Reynolds stress contribution which can include any type of turbulent spectrum is established in Sect. 3. In Sect. 4, the contribution of turbulent fluctuations of entropy is worked out. We find that it is the advection of the entropy turbulent fluctuation by the turbulent velocity field which yields the entropy source. An appendix shows that cross terms between Reynolds and entropic sources do not contribute.

Section 5 finally establishes a general formulation which can be used to investigate any type of turbulent spectrum. Finally, Sect. 6 discusses the complete formulation, its limitations and advantages.

\section{Turbulent stochastic excitation}

\subsection{The inhomogeneous wave equation}

In the basic hydrodynamical equations, we use the symbols $P, \rho, \boldsymbol{v}$ and $\boldsymbol{g}$ to denote respectively pressure, density, velocity and gravitational acceleration. Equilibrium quantities are represented with a subscript 0 . Each variable $f$, except for the velocity $\boldsymbol{v}$, is written as the sum of the equilibrium quantity, $f_{0}$ and a Eulerian fluctuation, $f_{1}, f=f_{0}+f_{1}$ and we retain terms which are linear and quadratic in the variables $P_{1}$ and $\rho_{1}$ and neglect the gravitational perturbation. Accordingly, one obtains for the perturbed momentum and continuity equations:

$$
\begin{array}{r}
\frac{\partial \rho \boldsymbol{v}}{\partial t}+\nabla:(\rho \boldsymbol{v} \boldsymbol{v})+\nabla P_{1}-\rho_{1} \boldsymbol{g}_{0}=0 \\
\frac{\partial \rho_{1}}{\partial t}+\nabla \cdot(\rho \boldsymbol{v})=0 .
\end{array}
$$

The perturbed equation of state in a Eulerian description is given by:

$P_{1}=c_{s}^{2} \rho_{1}+\alpha_{s} s_{1}+\alpha_{\rho \rho} \rho_{1}^{2}+\alpha_{s s} s_{1}^{2}+\alpha_{\rho s} \rho_{1} s_{1}$

where $s$ is the entropy, $\alpha_{s}=(\partial P / \partial s)_{\rho}, c_{s}=\Gamma_{1} P_{0} / \rho_{0}$ denotes the average sound speed, $\Gamma_{1}=(\partial \ln P / \partial \ln \rho)_{s}$ is the adiabatic exponent and $\alpha_{\rho \rho}, \alpha_{s s}$ and $\alpha_{\rho s}$ are the second partial derivatives of $P$ versus $s$ and $\rho$.

We assume adiabatic oscillations: the Lagrangian entropy fluctuations are therefore only due to turbulence. Lagrangian and Eulerian entropy fluctuations are related to each other by

$\frac{\mathrm{d} \delta s_{\mathrm{t}}}{\mathrm{d} t}=\frac{\partial s_{1}}{\partial t}+\boldsymbol{v} \cdot \boldsymbol{\nabla}\left(s_{0}+s_{1}\right)$

where $\delta s_{\mathrm{t}}$ is the turbulent Lagrangian entropy fluctuation. The subscript $t$ refers to turbulent quantities. For later use and with the help of Eq. (4), the time derivative of the Eulerian entropy fluctuation $s_{1}$ is written as

$\begin{aligned} \alpha_{s} \frac{\partial s_{1}}{\partial t}=\alpha_{s} \frac{\mathrm{d} \delta s_{\mathrm{t}}}{\mathrm{d} t}- & \alpha_{s} \boldsymbol{v} \cdot \nabla s_{0}-\nabla \cdot\left(\alpha_{s} s_{1} \boldsymbol{v}\right) \\ & +s_{1} \boldsymbol{v} \cdot \nabla \alpha_{s}+\alpha_{s} s_{1} \nabla \cdot \boldsymbol{v}\end{aligned}$

The velocity field $\boldsymbol{v}$ is split into a component due to the pulsational displacement $\delta \boldsymbol{r}_{\text {osc }}$ and a turbulent component $\boldsymbol{u}$ as $\boldsymbol{v}=\boldsymbol{v}_{\mathrm{osc}}+\boldsymbol{u}$.

Linearisation of Eqs. (1-3) yields for the velocity field, in the absence of turbulence $(\boldsymbol{u}=0)$, the homogeneous wave equation

$\left(\frac{\partial^{2}}{\partial t^{2}}-\boldsymbol{L}\right) \boldsymbol{v}=0$

with the linear wave operator:

$$
\begin{array}{r}
\boldsymbol{L}(\boldsymbol{X})=\frac{1}{\rho_{0}}\left[\nabla\left(c_{s}^{2} \nabla \cdot\left(\rho_{0} \boldsymbol{X}\right)+\alpha_{s} \boldsymbol{X} \cdot \boldsymbol{\nabla} s_{0}\right)\right. \\
\left.-\boldsymbol{g}_{0} \boldsymbol{\nabla} \cdot\left(\rho_{0} \boldsymbol{X}\right)\right] .
\end{array}
$$

With appropriate boundary conditions (Unno et al. 1989) one recovers the usual eigenvalue problem:

$$
\boldsymbol{L}(\boldsymbol{\xi}(\boldsymbol{r}))=-\omega_{0}^{2} \boldsymbol{\xi}(\boldsymbol{r})
$$


where $\omega_{0}$ is the oscillation frequency and $\boldsymbol{\xi}(\boldsymbol{r})$ is the adiabatic (real) displacement eigenvector.

In the presence of turbulence, the pulsational displacement and velocity are written in terms of the above adiabatic solution $\boldsymbol{\xi}(\boldsymbol{r}, t)$ and an instantaneous amplitude $A(t)$. Accordingly

$\boldsymbol{v}_{\mathrm{osc}}=\frac{\mathrm{d} \delta \boldsymbol{r}_{\mathrm{osc}}}{\mathrm{d} t}=\frac{1}{2}\left(-i \omega_{0} A(t) \boldsymbol{\xi}(\boldsymbol{r}) \mathrm{e}^{-i \omega_{0} t}+c c\right)$

where $c c$ means complex conjugate.

Differentiating Eq. (1) with respect to $t$, neglecting non-linear terms in $\boldsymbol{v}_{\text {osc }}$, assuming an incompressible turbulence $(\boldsymbol{\nabla} \cdot \boldsymbol{u}=0)$ and using Eqs. $(2,3,5)$ yields the inhomogeneous wave equation

$$
\begin{aligned}
& \rho_{0}\left(\frac{\partial^{2}}{\partial t^{2}}-\boldsymbol{L}\right)\left[\left(1+\frac{\rho_{1}}{\rho_{0}}\right) \boldsymbol{v}\right]+\mathcal{D}\left(\boldsymbol{v}_{\mathrm{osc}}\right)= \\
& \frac{\partial}{\partial t}\left(\boldsymbol{f}_{\mathrm{t}}+\nabla h_{\mathrm{t}}\right)
\end{aligned}
$$

with

$$
\boldsymbol{f}_{\mathrm{t}}=-\boldsymbol{\nabla}:\left(\rho_{0} \boldsymbol{u} \boldsymbol{u}\right)
$$

the turbulent Reynolds stress,

$$
\frac{\partial}{\partial t} \nabla h_{\mathrm{t}}=-\boldsymbol{\nabla}\left(\alpha_{s} \frac{\mathrm{d}}{\mathrm{d} t} \delta s_{\mathrm{t}}-\nabla \cdot\left(\alpha_{s} \boldsymbol{u} s_{1}\right)+s_{1} \boldsymbol{u} \cdot \boldsymbol{\nabla} \alpha_{s}\right)
$$

the source term due to the turbulent entropy fluctuations. The first term in the RHS of Eq. (12) is due to the Lagrangian entropy fluctuation which was considered by GMK and B92. The last two terms are due to the buoyancy force associated with the Eulerian entropy fluctuations. These terms contribute to the excitation as much as the Reynolds source term. The time derivative operator in the LHS of Eq. (12) in the definition of $h_{\mathrm{t}}$ is introduced for convenience.

The operator $\mathcal{D}$ involves both the turbulent velocity field $(\boldsymbol{u})$ and the pulsational velocity and is defined as

$$
\begin{aligned}
\mathcal{D}\left(\boldsymbol{v}_{\mathrm{osc}}\right)= & 2 \frac{\partial}{\partial t}\left(\boldsymbol{\nabla}: \rho \boldsymbol{u} \boldsymbol{v}_{\mathrm{osc}}\right)+\boldsymbol{\nabla}\left(\alpha_{s} \frac{\rho_{1}}{\rho_{0}} \boldsymbol{v}_{\mathrm{osc}} \cdot \boldsymbol{\nabla} s_{0}\right. \\
& -\boldsymbol{\nabla} \cdot\left(\alpha_{s} \boldsymbol{v}_{\mathrm{osc}} s_{1}\right)+s_{1} \boldsymbol{v}_{\mathrm{osc}} \cdot \boldsymbol{\nabla} \alpha_{s} \\
& \left.+\alpha_{s} s_{1} \boldsymbol{\nabla} \cdot \boldsymbol{v}_{\mathrm{osc}}-\left(\alpha_{\rho s} s_{1}+2 \alpha_{\rho \rho} \rho_{1}\right) \boldsymbol{\nabla} \cdot\left(\rho \boldsymbol{v}_{\mathrm{osc}}\right)\right) .
\end{aligned}
$$

As will be shown in the next section, this term contributes to the dynamical linear damping.

We assume a "free turbulence", i.e. the turbulent medium evolves freely and is not perturbed by the oscillations. The continuity equation is verified by the turbulent medium such that

$$
\frac{\partial \rho_{\mathrm{t}}}{\partial t}+\nabla \cdot\left(\left(\rho_{0}+\rho_{\mathrm{t}}\right) \boldsymbol{u}\right)=0 \text {. }
$$

As a consequence of neglecting the oscillation perturbations in higher order terms, the Eulerian fluctuations $s_{1}$ and $\rho_{1}$ can be seen as due only to the turbulence in evaluating the quantities $\nabla h_{\mathrm{t}}$ and $\mathcal{D}$. We therefore substitute $\rho_{1}$ (resp. $\left.s_{1}\right)$ by $\rho_{\mathrm{t}}$ (resp. $s_{\mathrm{t}}$ ) in Eqs. $((12),(14))$.
It can easily be shown that the additional terms appearing in the RHS of Eq. (10) are of order $M_{\mathrm{t}}^{2}$, where $M_{\mathrm{t}}$ is the turbulent Mach number, compared with the Reynolds source term (see also GK). As $M_{\mathrm{t}}$ is small in the solar convection zone $\left(M_{\mathrm{t}} \lesssim 0.3\right)$, these additional terms have been neglected.

The wave operator $\left(\partial^{2} / \partial t^{2}-\boldsymbol{L}\right)$ acting on the turbulent velocity field $\boldsymbol{u}$ in Eq. (10) gives rise to contributions which are either negligible compared with the Reynolds source term or of the same order. In this last case, the associated source term does not contribute to the wave excitation because it is linear in terms of the turbulent fluctuations (see also Sect. 4.1). Therefore, in the LHS of Eq. (10), $\boldsymbol{v}$ can be replaced by $\boldsymbol{v}_{\text {osc }}$.

In deriving Eq. (9), the time variation of the amplitude $A$ is neglected since we assume, as in the solar case, that the eddy time correlation is of the order of a few minutes in the excitation region and the associated angular frequency is comparable to $\omega_{0}(\sim 3 \mathrm{mHz})$, which is much larger than the oscillation damping rate $\eta(\sim 100 \mu \mathrm{Hz})$.

\subsection{Mean square amplitude}

Substituting Eq. (9) into Eq. (10), gives, with the help of Eq. (6),

$$
\begin{gathered}
2 \omega_{0}^{2} \frac{\mathrm{d} A}{\mathrm{~d} t} \rho_{0} \boldsymbol{\xi}+i \omega_{0} \mathcal{D}(A \boldsymbol{\xi}) \\
+i \omega_{0} \rho_{0}\left(\frac{\partial^{2}}{\partial t^{2}}-\boldsymbol{L}\right)\left[\frac{\rho_{\mathrm{t}}}{\rho_{0}} A \boldsymbol{\xi}\right]=\frac{\partial \mathcal{S}}{\partial t}
\end{gathered}
$$

with

$\mathcal{S} \equiv-\left(f_{\mathrm{t}}+\nabla h_{\mathrm{t}}\right)$

As previously mentioned, periods of oscillations are much shorter than their lifetime $(\sim 1 / \eta)$ such that $\omega_{0} \gg \eta$ and thus $|\mathrm{d} \ln A / \mathrm{d} t| \ll \omega_{0}$. Therefore the second derivative of $A$ arising in Eq. (15) has been discarded. Multiplying Eq. (15) by $\rho_{0} \boldsymbol{\xi}^{*}(\boldsymbol{r}, t)$ and integrating over the stellar volume gives

$\frac{\mathrm{d} A}{\mathrm{~d} t}+\Delta \sigma A=\frac{1}{2 \omega_{0}^{2} I} \int \mathrm{d}^{3} x \boldsymbol{\xi}^{*} \cdot \frac{\partial \mathcal{S}}{\partial t}$,

where we have defined the complex quantity $\Delta \sigma=i \Delta \omega+$ $\eta_{D}$ which comes from the contribution of the operator $\mathcal{D}(\boldsymbol{\xi})$. This expression is linear in the eigenfunction $\xi$, hence $\Delta \omega$ corresponds to a "dynamical" shift of the oscillation frequency due to turbulence. This shift is expected to be smaller or of the order of non-adiabatic frequency shifts, which are not considered here. The dynamical shift, $\Delta \omega$, will therefore be neglected. The real part, $\eta_{D}$, contributes to the dynamical damping. As in B92 and GK, $\eta_{D}$ is replaced in Eq. (17) by the global damping rate $\eta$ in order to take into account all damping processes. The solution of Eq. (17) after integration by parts over the time is given by:

$A(t)=\frac{i \mathrm{e}^{-\eta t}}{2 \omega_{0} I} \int_{-\infty}^{t} \mathrm{~d} t^{\prime} \int \mathrm{d}^{3} x \mathrm{e}^{\left(\eta+i \omega_{0}\right) t^{\prime}} \boldsymbol{\xi}(\boldsymbol{x}) \cdot \mathcal{S}\left(\boldsymbol{x}, t^{\prime}\right)$ 
and for the square amplitude:

$$
\begin{aligned}
|A|^{2}(t) & =\frac{\mathrm{e}^{-2 \eta t}}{4\left(\omega_{0} I\right)^{2}} \int_{-\infty}^{t} \mathrm{~d} t_{1} \mathrm{~d} t_{2} \int \mathrm{d}^{3} r_{1} \mathrm{~d}^{3} r_{2} \mathrm{e}^{\sigma\left(t_{1}, t_{2}\right)} \\
& \times\left(\boldsymbol{\xi}\left(\boldsymbol{r}_{1}\right) \cdot \mathcal{S}\left(\boldsymbol{r}_{\mathbf{1}}, \boldsymbol{t}_{\mathbf{1}}\right)\right)\left(\boldsymbol{\xi}\left(\boldsymbol{r}_{\mathbf{2}}\right) \cdot \mathcal{S}^{*}\left(\boldsymbol{r}_{\mathbf{2}}, \boldsymbol{t}_{\mathbf{2}}\right)\right)
\end{aligned}
$$

with $\sigma\left(t_{1}, t_{2}\right)=\eta\left(t_{1}+t_{2}\right)+i \omega_{0}\left(t_{1}-t_{2}\right)$. Spatial integrations are performed over the whole stellar envelope. For convenience we define the following coordinates

$$
\begin{array}{ll}
\boldsymbol{x}_{0}=\frac{\boldsymbol{r}_{2}+\boldsymbol{r}_{1}}{2} & t_{0}=\frac{t_{1}+t_{2}}{2} \\
\boldsymbol{r}=\boldsymbol{r}_{2}-\boldsymbol{r}_{1} & \tau=t_{2}-t_{1}
\end{array}
$$

$\boldsymbol{x}_{0}$ and $t_{0}$ are the average time-space position where the stochastic excitation is integrated whereas $\boldsymbol{r}$ and $\tau$ are related to the local turbulence. $\tau$ corresponds to the fast time scale associated with the eddy lifetime and $t_{0}$ to the slow time scale associated with the oscillation growth rate $(\eta)$. In the following, $\nabla_{0}$ is the large-scale derivative associated with $\boldsymbol{x}_{0}, \boldsymbol{\nabla}_{\boldsymbol{r}}$ is the small-scale one associated with $\boldsymbol{r}$ and the derivative operators $\nabla_{1}$ and $\nabla_{2}$ are associated with $\boldsymbol{r}_{1}$ and $\boldsymbol{r}_{2}$ respectively. The mean square amplitude can be rewritten in terms of the new coordinates as

$$
\begin{aligned}
\left\langle|A|^{2}(t)\right\rangle= & \frac{1}{4\left(\omega_{0} I\right)^{2}} \int_{-\infty}^{t} \mathrm{~d} t_{0} \mathrm{e}^{2 \eta\left(t_{0}-t\right)} \int_{2\left(t_{0}-t\right)}^{2\left(t-t_{0}\right)} \mathrm{d} \tau \\
& \times \int \mathrm{d}^{3} x_{0} \mathrm{~d}^{3} r \mathrm{e}^{-i \omega_{0} \tau} \\
\times & \left\langle\boldsymbol{\xi} \cdot \mathcal{S}\left[\boldsymbol{x}_{0}-\frac{\boldsymbol{r}}{2}, t_{0}-\frac{\tau}{2}\right] \boldsymbol{\xi} \cdot \mathcal{S}^{*}\left[\boldsymbol{x}_{0}+\frac{\boldsymbol{r}}{2}, t_{0}+\frac{\tau}{2}\right]\right\rangle .
\end{aligned}
$$

The operator $\langle$.$\rangle denotes the statistical average performed$ on an infinite number of independent realizations.

In the excitation region the eddy lifetime is much smaller than the oscillation lifetime $(\sim 1 / \eta)$ such that the integration over $\tau$ can be extended to infinity. We assume a stationary turbulence, therefore the source term $\mathcal{S}$ is invariant over time $t_{0}$. Integration over $t_{0}$ in Eq. (21) and using the definition of $\mathcal{S}$ in Eq. (16) yields:

$$
\left\langle|A|^{2}\right\rangle=\frac{1}{8 \eta\left(\omega_{0} I\right)^{2}}\left(C_{\mathrm{R}}^{2}+C_{\mathrm{S}}^{2}\right)
$$

with

$$
\begin{aligned}
C_{\mathrm{R}}^{2}= & \int \mathrm{d}^{3} x_{0} \int_{-\infty}^{+\infty} \mathrm{d} \tau \mathrm{e}^{-i \omega_{0} \tau} \int \mathrm{d}^{3} r \\
& \times\left\langle\left(\rho_{0} u_{j} u_{i} \nabla_{1}^{j} \xi^{i}\right)_{1}\left(\rho_{0} u_{j} u_{i} \nabla_{2}^{j} \xi^{i}\right)_{2}\right\rangle
\end{aligned}
$$

the turbulent Reynolds stress contribution,

$$
\begin{aligned}
C_{\mathrm{S}}^{2}= & \int \mathrm{d}^{3} x_{0} \int_{-\infty}^{+\infty} \mathrm{d} \tau \mathrm{e}^{-i \omega_{0} \tau} \int \mathrm{d}^{3} r \\
& \times\left\langle\left(h_{\mathrm{t}} \boldsymbol{\nabla} \cdot \boldsymbol{\xi}\right)_{1}\left(h_{\mathrm{t}} \boldsymbol{\nabla} \cdot \boldsymbol{\xi}\right)_{2}\right\rangle
\end{aligned}
$$

the entropy contribution. Subscripts 1 and 2 are the values taken at the spatial and temporal positions $\left[\boldsymbol{x}_{0}-\frac{\boldsymbol{r}}{2},-\frac{\tau}{2}\right]$ and $\left[\boldsymbol{x}_{0}+\frac{r}{2}, \frac{\tau}{2}\right]$ respectively. We adopt the Einstein convention of summation upon repeated indices. Hereafter all time integrations over $\tau$ are understood to be performed in the range $]-\infty,+\infty[$. Note that the crossing term between the Reynolds source term and the entropy source term does not contribute to the excitation (see Appendix A).

\section{Reynolds stress contribution}

In the derivation of Eqs. (23) and (24) integrations by parts have been performed in order for the gradient to act on the eigenfunction instead of turbulent quantities. We next suppose that the terms $\left(\rho_{0} \nabla_{1}^{i} \xi^{j}\right)$ and $\left(\rho_{0} \nabla_{2}^{i} \xi^{j}\right)$ in Eq. (23) do not change on the length scale of the eddies. This implies that $\nabla_{r}^{i} \xi^{j} \simeq 0$. Validity of this assumption will be justified a posteriori below. Consequently, the Reynolds stress contribution can be expressed as:

$$
\begin{aligned}
C_{\mathrm{R}}^{2}= & \int \mathrm{d}^{3} x_{0} \rho_{0}^{2} \nabla_{0}^{i} \xi^{j} \nabla_{0}^{l} \xi^{m} \\
& \times \int \mathrm{d} \tau \mathrm{d}^{3} r \mathrm{e}^{-i \omega_{0} \tau}\left\langle u_{i}^{\prime} u_{j}^{\prime} u_{l}^{\prime \prime} u_{m}^{\prime \prime}\right\rangle
\end{aligned}
$$

where the following notations have been used:

$\boldsymbol{u}^{\prime}=\boldsymbol{u}\left(\boldsymbol{x}_{0}-\frac{\boldsymbol{r}}{2}, t_{0}-\frac{\tau}{2}\right) \quad \boldsymbol{u}^{\prime \prime}=\boldsymbol{u}\left(\boldsymbol{x}_{0}+\frac{\boldsymbol{r}}{2}, t_{0}+\frac{\tau}{2}\right)$.

The Quasi-Normal Approximation (Lesieur 1997, Chap. VII-2, QNA hereafter) reduces the fourth-order velocity correlations as follows:

$$
\begin{aligned}
\left\langle u_{i}^{\prime} u_{j}^{\prime} u_{l}^{\prime \prime} u_{m}^{\prime \prime}\right\rangle=\left\langle u_{i}^{\prime} u_{j}^{\prime}\right\rangle\left\langle u_{l}^{\prime \prime} u_{m}^{\prime \prime}\right\rangle & +\left\langle u_{i}^{\prime} u_{l}^{\prime \prime}\right\rangle\left\langle u_{j}^{\prime} u_{m}^{\prime \prime}\right\rangle \\
& +\left\langle u_{i}^{\prime} u_{m}^{\prime \prime}\right\rangle\left\langle u_{j}^{\prime} u_{l}^{\prime \prime}\right\rangle .
\end{aligned}
$$

This approximation remains strictly valid for normally distributed fluctuating quantities. As shown by Kraichnan (1957) and Stein (1967) neglected terms can be large and therefore not negligible. Here the QNA approximation is nevertheless assumed valid as it is found justified when considering 3D simulations of the solar convection zone (Samadi 2000, work in progress).

We denote $\phi_{i j}(\boldsymbol{k}, \omega)$ as the well known Fourier transform of the second-order velocity correlations $\left\langle u_{i}^{\prime} u_{j}^{\prime \prime}\right\rangle$ (e.g. Stein 1967). For a stationary, incompressible, homogeneous and isotropic turbulence, the Fourier transform of the velocity correlation has the form (Batchelor 1970):

$\phi_{i j}(\boldsymbol{k}, \omega)=\frac{E(k, \omega)}{4 \pi k^{2}}\left(\delta_{i j}-\frac{k_{i} k_{j}}{k^{2}}\right)$

where $E(k, \omega)$ is the turbulent kinetic energy spectrum and $\delta_{i j}$ is the Kroenecker tensor. Following Stein (1967), the velocity energy spectrum $E(k, \omega)$ is written as

$E(k, \omega)=E(k) \chi_{k}(\omega)$.

Stein (1967) and Musielak et al. (1994) suggest several forms for the frequency factor. The Gaussian function is the simplest choice and is defined as

$\chi_{k}(\omega)=\frac{1}{\omega_{k} \sqrt{\pi}} \mathrm{e}^{-\left(\omega / \omega_{k}\right)^{2}}$

The time spectrum is therefore normalized such that (Tennekes \& Lumley 1982, Chap. 8.1)

$\int_{-\infty}^{+\infty} \mathrm{d} \omega \chi_{k}(\omega)=1$. 
One obtains from Eq. (27):

$$
\begin{aligned}
\int \mathrm{d} \tau & \mathrm{d}^{3} r \mathrm{e}^{-i \omega_{0} \tau}\left\langle u_{i}^{\prime} u_{j}^{\prime} u_{l}^{\prime \prime} u_{m}^{\prime \prime}\right\rangle=(2 \pi)^{4} \int \mathrm{d} \omega \mathrm{d}^{3} k \\
\times & {\left[\phi_{i l}\left(\boldsymbol{k}, \omega_{0}\right) \phi_{j m}(\boldsymbol{k}, \omega)+\phi_{i m}\left(\boldsymbol{k}, \omega_{0}\right) \phi_{j l}(\boldsymbol{k}, \omega)\right] . }
\end{aligned}
$$

The first term in the RHS of Eq. (27) when inserted into Eq. (32) gives no contribution. With the help of Eqs. (31) and (32), the Reynolds stress contribution can be written as

$$
\begin{aligned}
C_{\mathrm{R}}^{2}= & \pi^{2} \int \mathrm{d}^{3} x_{0}\left(\rho_{0}^{2} \nabla_{0}^{i} \xi^{j} \nabla_{0}^{l} \xi^{m}\right) \int \mathrm{d}^{3} k \\
& \times\left(T_{i j l m}+T_{i j m l}\right) \frac{E^{2}(k)}{k^{4}} \chi_{k}\left(\omega_{0}\right)
\end{aligned}
$$

where

$T_{i j l m}=\left(\delta_{i l}-\frac{k_{i} k_{l}}{k^{2}}\right)\left(\delta_{j m}-\frac{k_{j} k_{m}}{k^{2}}\right)$.

Excitation by convection takes place at the top of the convection zone. In this region, eigenvectors of acoustic modes with high radial order can be considered essentially as radial and propagating in a plane-parallel manner. Hence Eq. (33) becomes (see Appendix C)

$C_{\mathrm{R}}^{2}=4 \pi^{3} \mathcal{G} \int_{0}^{M} \mathrm{~d} m \rho_{0}\left(\frac{\mathrm{d} \xi_{r}}{\mathrm{~d} r}\right)^{2} \int_{0}^{\infty} \mathrm{d} k \frac{E^{2}(k)}{k^{2}} \chi_{k}\left(\omega_{0}\right)$

where $\mathcal{G}$ is an anisotropic factor similar to Gough's (1977) one, as defined in Eq. (C.6). Because of the term $\chi_{k}\left(\omega_{0}\right)$, eddies with $\omega_{k} \gtrsim \omega_{0}$ contribute the most to the integration over $k$, i.e. to mode excitation. This is in agreement with Goldreich \& Kumar (1990), who state that acoustic emission arises from eddies with $\omega_{0} \tau_{k} \lesssim 1$. We have

$\frac{\omega_{k}}{\omega_{0}} \approx \frac{k u_{k}}{k_{\mathrm{osc}} c_{s}} \gtrsim \approx \frac{k}{k_{\mathrm{osc}}} M_{\mathrm{t}} \gtrsim 1$

where $k_{\text {osc }}$ is the wavenumber of the mode, $u_{k}$ the velocity of an eddy with wavenumber $k$ and $M_{\mathrm{t}}$ the turbulent Mach number, which is small. Eddies with $k \gg k_{\text {osc }}$ are those which contribute the most to modal excitation. The oscillation and the contributive eddies are then well decoupled. Moreover, the stratification does not affect the turbulent emission (Goldreich \& Kumar 1990). The above comments altogether justify the assumption - at the beginning of this section - that $\left(\rho_{0}^{2} \nabla_{1}^{i} \xi^{j} \nabla_{2}^{k} \xi^{l}\right)$ remains constant over the length scale of the contributive eddies. These conclusions also justify the use of a homogeneous turbulence because the stratification and the oscillations have a characteristic scale length much larger than the contributive eddies.

\section{Contribution of entropy fluctuations}

As in Sect. 3 we use $\nabla_{r}^{i} \xi^{j} \simeq 0$ in Eq. (24) to obtain:

$C_{\mathrm{S}}^{2}=\int \mathrm{d}^{3} x_{0}\left(\nabla_{0}^{i} \xi_{i}\right)^{2} \int \mathrm{d} \tau \mathrm{e}^{-i \omega_{0} \tau} \int \mathrm{d}^{3} r\left\langle h_{\mathrm{t}}^{\prime} h_{\mathrm{t}}^{\prime \prime}\right\rangle$ where

$h_{\mathrm{t}}^{\prime}=h_{\mathrm{t}}\left(\boldsymbol{x}_{0}-\frac{\boldsymbol{r}}{2},-\frac{\tau}{2}\right)$ and $h_{\mathrm{t}}^{\prime \prime}=h_{\mathrm{t}}\left(\boldsymbol{x}_{0}+\frac{\boldsymbol{r}}{2},+\frac{\tau}{2}\right)$

Integration over the time of the first term in the RHS of Eq. (12) gives

$h_{\mathrm{t}}(\boldsymbol{x}, t)=-\alpha_{s} \delta s_{\mathrm{t}}+q_{\mathrm{t}}(\boldsymbol{x}, t)$

with

$q_{\mathrm{t}}(\boldsymbol{x}, t) \equiv \int_{-\infty}^{t} \mathrm{~d} t^{\prime}\left(\boldsymbol{\nabla} \cdot\left(\alpha_{s} \boldsymbol{u} s_{\mathrm{t}}\right)-s_{\mathrm{t}} \boldsymbol{u} \cdot \nabla \alpha_{s}\right)$.

Contribution from the crossing term between the term $\alpha_{s} \delta s_{\mathrm{t}}$ (the linear term) and the term $q_{\mathrm{t}}$ (the non linear term), i.e. $\left\langle\alpha_{s} \delta s_{\mathrm{t}}^{\prime} q_{\mathrm{t}}^{\prime \prime}\right\rangle$, vanishes (cf. Appendix A). We are left with

$$
\begin{aligned}
C_{\mathrm{S}}^{2}= & \int \mathrm{d}^{3} x_{0}\left(\nabla_{0}^{i} \xi_{i}\right)^{2} \int \mathrm{d} \tau \mathrm{e}^{-i \omega_{0} \tau} \int \mathrm{d}^{3} r \\
& \times\left[\left\langle\left(\alpha_{s} \delta s_{\mathrm{t}}^{\prime}\right)\left(\alpha_{s} \delta s_{\mathrm{t}}^{\prime \prime}\right)\right\rangle+\left\langle q_{\mathrm{t}}^{\prime} q_{\mathrm{t}}^{\prime \prime}\right\rangle\right] .
\end{aligned}
$$

\subsection{Contribution of the linear term}

We consider the contribution of the Lagrangian entropy fluctuations (i.e. the first term in the RHS of Eq. (40)).

In the Boussines approximation, the entropy fluctuation $s_{\mathrm{t}}$ is related to the temperature fluctuation $T_{\mathrm{t}}$ as

$s_{\mathrm{t}}=\left(\frac{\partial s}{\partial T}\right)_{\mathrm{P}} T_{\mathrm{t}}$.

In a free turbulent medium the Eulerian temperature fluctuation acts as a passive scalar (Tennekes \& Lumley 1982; Lesieur 1997, Chap. VI-10). Thus one can expect that the Eulerian entropy turbulent fluctuation acts as a passive scalar.

Let $\phi_{s}(\boldsymbol{k}, \omega)$ be the Fourier transform of the correlation product of the Eulerian entropy fluctuation. For any passive scalar, one has the relation (Lesieur 1997, Chap. V-10)

$\phi_{s}(\boldsymbol{k}, \omega)=\frac{E_{\mathrm{S}}(k, \omega)}{2 \pi k^{2}}$

where the scalar spectrum $E_{\mathrm{s}}(k, \omega)$ is related to the scalar variance as (Lesieur 1997, Chap. V-10)

$\frac{1}{2}\left\langle s_{\mathrm{t}}^{2}\left(\boldsymbol{x}_{0}, \omega\right)\right\rangle=\int_{0}^{\infty} \mathrm{d} k E_{\mathrm{s}}(k, \omega)$.

Because the Lagrangian entropy fluctuation acts as a passive scalar as well, $\delta s_{\mathrm{t}}$ scales as $s_{\mathrm{t}}$. The contribution of the Lagrangian entropy fluctuation is therefore proportional to

$\int_{-\infty}^{+\infty} \mathrm{d} \tau \int \mathrm{d}^{3} r \mathrm{e}^{-i \omega_{0} \tau}\left\langle s^{\prime} s^{\prime \prime}\right\rangle=(2 \pi)^{4} \phi_{s}\left(\mathbf{0}, \omega_{0}\right)$,

where the following notations have been defined:

$s^{\prime}=s_{\mathrm{t}}\left(\boldsymbol{x}_{0}-\frac{\boldsymbol{r}}{2}, t_{0}-\frac{\tau}{2}\right) \quad s^{\prime \prime}=s_{\mathrm{t}}\left(\boldsymbol{x}_{0}+\frac{\boldsymbol{r}}{2}, t_{0}+\frac{\tau}{2}\right)$ 
As was done with the kinetic energy spectrum, the scalar energy spectrum $E_{\mathrm{s}}(k, \omega)$ is decomposed such that

$E_{\mathrm{s}}(k, \omega)=E_{\mathrm{s}}(k) \chi_{k}(\omega)$

with $\chi_{k}(\omega)$ the same frequency-dependent factor as in Sect. 3. Let $\chi_{k}(\omega)$ take the Gaussian form: as $\omega_{k} \propto k u_{k}$ we have $\chi_{k}\left(\omega_{0}\right)=0$ for $k=0$. As $E_{\mathrm{s}}(k)$ increases as $k^{2}$ in the vicinity of $k=0$ (Tennekes 1975; Tennekes \& Lumley 1982, Chap. 8.6), the quantity $\phi_{\mathbf{s}}\left(\mathbf{0}, \omega_{0}\right)$ vanishes and so does the linear term contribution. We emphasize that in the context of the approximation presented in Eq. (46), no assumption has been made regarding the behavior of the entropy energy spectrum $E_{\mathrm{s}}(\boldsymbol{k})$. Hence, this result remains valid for any scalar spectrum and for the velocity energy spectrum.

This result may also be explained as follows: in term of mode excitation, the linear entropy source term acts as a second-order correlation product $\left\langle s^{\prime} s^{\prime \prime}\right\rangle$. Turbulence and oscillation are coupled through the phase term $\mathrm{e}^{-i \omega_{0} \tau}$ and through the turbulent time spectrum $\chi_{k}(\omega)$, which is the frequency-dependent component of $\left\langle s^{\prime} s^{\prime \prime}\right\rangle$ in the Fourier space. Therefore coupling between turbulence and oscillation occurs at frequencies close to the oscillation frequency $\omega_{0}$ and thus involves eddies of wavenumber $k \gtrsim k_{\text {osc }} M_{\mathrm{t}}^{-1}$ according to Eq. (35). On the other hand, the spatial component of $\left\langle s^{\prime} s^{\prime \prime}\right\rangle$ in the Fourier space favors eddies with the largest size $(k \rightarrow 0)$. These two opposite effects clearly are incompatible and lead to vanishing of the entropy fluctuation contribution.

This does not happen for the contribution of the Reynolds source term, which involves the fourth-order velocity correlation product. According to the QNA this term can be decomposed in terms of a product of two second-order velocity correlations. Coupling with the oscillation then becomes non-linear and leads to an effective non-zero contribution. Thus, only non-linear terms can contribute to mode excitation while linear terms do not. This may be considered as a general result and justifies neglect of several source terms in Sect. 2.2.

\subsection{Contribution of the non-linear terms}

As the linear term does not contribute to the acoustic emission, Eq. (40) becomes, with Eq. (39),

$$
\begin{aligned}
C_{\mathrm{S}}^{2}= & \int \mathrm{d}^{3} x_{0}\left(\boldsymbol{\nabla}_{0} \cdot \boldsymbol{\xi}\right)^{2} \int \mathrm{d} \tau \mathrm{e}^{-i \omega_{0} \tau} \int \mathrm{d}^{3} r \\
& \times \int_{-\infty}^{\tau / 2} \mathrm{~d} t_{3} \int_{-\infty}^{-\tau / 2} \mathrm{~d} t_{4} \\
& \times\left\{\boldsymbol{\nabla}_{1} \alpha_{s} \cdot\left\langle\left(s_{\mathrm{t}} \boldsymbol{u}\right)_{3}\left(s_{\mathrm{t}} \boldsymbol{u}\right)_{4}\right\rangle \cdot \nabla_{2} \alpha_{s}\right. \\
& -2 \boldsymbol{\nabla}_{1} \alpha_{s} \cdot\left\langle\left(s_{\mathrm{t}} \boldsymbol{u}\right)_{3} \boldsymbol{\nabla}_{2} \cdot\left(\alpha_{s} s_{\mathrm{t}} \boldsymbol{u}\right)_{4}\right\rangle \\
& \left.+\left\langle\boldsymbol{\nabla}_{1} \cdot\left(\alpha_{s} s_{\mathrm{t}} \boldsymbol{u}\right)_{3} \boldsymbol{\nabla}_{2} \cdot\left(\alpha_{s} s_{\mathrm{t}} \boldsymbol{u}\right)_{4}\right\rangle\right\}
\end{aligned}
$$

where subscripts 3 and 4 refer to evaluations at positions $\left[\boldsymbol{x}_{0}-\boldsymbol{r} / 2, t_{3}\right]$ and $\left[\boldsymbol{x}_{0}+\boldsymbol{r} / 2, t_{4}\right]$ respectively. Similar way to the Reynolds contribution, we have $\nabla_{1}^{i} \alpha_{s} \simeq \nabla_{2}^{i} \alpha_{s} \simeq$ $\nabla_{0}^{i} \alpha_{s}$. We use an integration by parts and the derivative operators $\nabla_{1}$ and $\nabla_{2}$ are replaced by the large-scale gradient $\nabla_{0}$. This finally gives

$$
\begin{aligned}
C_{\mathrm{S}}^{2}= & \int \mathrm{d}^{3} x_{0}\left(\alpha_{s} \nabla_{0} \cdot \boldsymbol{\xi}\right)^{2} g^{i j} \int \mathrm{d} \tau \mathrm{e}^{-i \omega_{0} \tau} \int \mathrm{d}^{3} r \\
& \times \int_{-\infty}^{\tau / 2} \mathrm{~d} t_{3} \int_{-\infty}^{-\tau / 2} \mathrm{~d} t_{4}\left\langle\left(s_{\mathrm{t}} u_{i}\right)_{3}\left(s_{\mathrm{t}} u_{j}\right)_{4}\right\rangle
\end{aligned}
$$

with

$$
\begin{aligned}
g^{i j}= & \nabla_{0}^{i}\left(\ln \left|\alpha_{s}\right|\right) \nabla_{0}^{j}\left(\ln \left|\alpha_{s}\right|\right) \\
& -2 \nabla_{0}^{i}\left(\ln \left|\alpha_{s}\right|\right) \nabla_{0}^{j}\left(\ln \left|\nabla_{0} . \boldsymbol{\xi}\right|\right) \\
& +\nabla_{0}^{i}\left(\ln \left|\nabla_{0} . \boldsymbol{\xi}\right|\right) \nabla_{0}^{j}\left(\ln \left|\nabla_{0} . \boldsymbol{\xi}\right|\right)
\end{aligned}
$$

Again, we consider the gradient of the stratification as radial and a plane parallel approximation in the excitation region. One obtains (see Appendices B and C)

$$
\begin{aligned}
C_{\mathrm{S}}^{2}= & \frac{4 \pi^{3} \mathcal{H}}{\omega_{0}^{2}} \int \mathrm{d}^{3} x_{0}\left(\alpha_{s} \frac{\mathrm{d} \xi_{r}}{\mathrm{~d} r}\right)^{2} g_{r} \\
& \times \int \mathrm{d} k \frac{E_{\mathrm{s}}(k) E(k)}{k^{2}} \int_{-\infty}^{+\infty} \mathrm{d} \omega \chi_{k}\left(\omega_{0}+\omega\right) \chi_{k}(\omega)
\end{aligned}
$$

with

$g_{r}=\frac{1}{r^{2}}\left(\frac{\mathrm{d}}{\mathrm{d} \ln r} \ln \left|\alpha_{s}\right|-\frac{\mathrm{d}}{\mathrm{d} \ln r} \ln \left|\frac{\mathrm{d}}{\mathrm{d} r} \xi_{r}\right|\right)^{2}$

and $\mathcal{H}$ an anisotropic factor similar to Gough's anisotropic factor (see Appendix C).

The Mixing-Length Theory (hereafter MLT BöhmVitense 1958; Cox 1968; Gough 1977) provides an estimate of the vertical velocity of the convective flow. The corresponding kinetic energy is transferred to smaller scales through the turbulent cascade. The kinetic energy spectrum $E(k)$ is normalized as

$\frac{1}{2}\left\langle u^{2}\left(\boldsymbol{x}_{0}\right)\right\rangle \equiv \int_{0}^{\infty} \mathrm{d} k E(k)=\frac{1}{2} \Phi w^{2}=\frac{3}{2} u_{0}^{2}$

where $w$ is an estimate for the vertical convective velocity and $\Phi$ is a factor introduced by Gough (1977) to take into account anisotropy effects (Eq. (C.6)). $u_{0}$ in the RHS of Eq. (52) has been introduced for convenience.

The MLT provides a relation between the temperature fluctuations and the vertical convective velocity $w$ (Gough 1977):

$w^{2}=\frac{g \Lambda \delta}{2 \Phi T}\left\langle T_{\mathrm{t}}^{2}\right\rangle^{1 / 2}$

where $\delta=\left(\frac{\partial \ln \rho_{0}}{\partial \ln T}\right)_{\mathrm{P}}$. Thus Eqs. $(41,53)$ provide an estimate of the entropy scalar variance

$\tilde{s}^{2} \equiv\left\langle s_{\mathrm{t}}^{2}\left(\boldsymbol{x}_{0}\right)\right\rangle=\left(\frac{2 \Phi C_{\mathrm{P}}}{g \Lambda \delta}\right)^{2} w^{4}$.

This enables us to normalize the entropy spectrum $E_{\mathbf{s}}(k)$

$\frac{1}{2}\left\langle s_{\mathrm{t}}^{2}\left(\boldsymbol{x}_{0}\right)\right\rangle=\int_{0}^{\infty} \mathrm{d} k E_{\mathrm{s}}(k)=\frac{1}{2} \tilde{s}^{2}$. 
Following GMK we define $\mathcal{R}$ as

$\mathcal{R} \equiv \frac{\alpha_{s} \tilde{s}}{\rho_{0} u_{0}^{2}}=\frac{6}{\alpha_{\mathrm{c}}} \Gamma_{1}$,

where $\alpha_{\mathrm{c}}$ is the mixing-length parameter defined as usual by $\Lambda=\alpha_{\mathrm{c}} H_{\mathrm{p}}$ with $\Lambda$ being the mixing-length and $H_{\mathrm{p}}$ the pressure scale height and $\Gamma_{1}$ is the usual adiabatic index. The quantity $\mathcal{R}^{2}$ roughly measures the ratio of the excitation by entropy fluctuations to that by fluctuations of Reynolds stresses.

The entropy contribution can then be written as:

$$
\begin{aligned}
C_{\mathrm{S}}^{2}= & 4 \pi^{3} \mathcal{H} \int_{0}^{M} \mathrm{~d} m \rho_{0} u_{0}^{4} \mathcal{R}^{2} \mathcal{F}^{2}\left(\frac{\mathrm{d} \xi_{r}}{\mathrm{~d} r}\right)^{2}\left(\frac{u_{0}}{\Lambda \omega_{0}}\right)^{2} \\
& \times \int \frac{\mathrm{d} k}{k^{2}} \frac{E_{\mathrm{S}}(k) E(k)}{\tilde{s}^{2} u_{0}^{2}} \int_{-\infty}^{+\infty} \mathrm{d} \omega \chi_{k}\left(\omega_{0}+\omega\right) \chi_{k}(\omega)
\end{aligned}
$$

with $\mathcal{F}^{2} \equiv \Lambda^{2} g_{r}$.

\section{Complete formulation}

With Eqs. (34) and (57), the oscillation amplitude Eq. (22) is rewritten as

$$
\begin{array}{r}
\left\langle|A|^{2}\right\rangle=\frac{\pi^{3}}{2 \eta\left(\omega_{0} I\right)^{2}} \int_{0}^{M} \mathrm{~d} m \rho_{0} u_{0}^{4}\left(\frac{\mathrm{d} \xi_{r}}{\mathrm{~d} r}\right)^{2} \\
\times \int_{0}^{\infty} \frac{\mathrm{d} k}{k^{2}} \frac{E(k)}{u_{0}^{2}} F\left(k, \omega_{0}\right)
\end{array}
$$

where

$$
\begin{aligned}
F\left(k, \omega_{0}\right) \equiv & \mathcal{G} \frac{E(k)}{u_{0}^{2}} \chi_{k}\left(\omega_{0}\right)+\mathcal{H} \mathcal{R}^{2} \mathcal{F}^{2} \frac{E_{\mathrm{s}}(k)}{\tilde{s}^{2}}\left(\frac{u_{0}}{\Lambda \omega_{0}}\right)^{2} \\
& \times \int_{-\infty}^{+\infty} \mathrm{d} \omega \chi_{k}\left(\omega_{0}+\omega\right) \chi_{k}(\omega)
\end{aligned}
$$

where $\mathcal{G}$ and $\mathcal{H}$ are given in Appendix C.

Results in Samadi et al. (2001a) for the solar case and in Samadi et al. (2001c) for Procyon are based on the above general expression for the mean square amplitude. In order to compare with results of previous works, we next consider specific temporal and energy spectra, namely the Gaussian time spectrum and the Kolmogorov spectrum.

\subsection{Gaussian time spectrum}

Let consider the Gaussian time spectrum given by Eq. (30). This time spectrum corresponds in the time space to a Gaussian function where linewidth is equal to $2 \tau_{k}$ and $\tau_{k}$ is the characteristic time correlation length of an eddy of wavenumber $k$. Hence $\omega_{k}$ and $\tau_{k}$ are related to each other as

$\omega_{k}=\frac{2}{\tau_{k}}$.

As in B92 we define $\tau_{k}$ as

$\tau_{k} \equiv \frac{\lambda}{k u_{k}}$ where the velocity $u_{k}$ of the eddy with wave number $k$ is related to the kinetic energy spectrum $E(k)$ as (Stein 1967)

$u_{k}^{2}=\int_{k}^{2 k} \mathrm{~d} k E(k)$.

The factor $\lambda$ in Eq. (61) is introduced in order to gauge the definitions of $\tau_{k}$ and $u_{k}$ which involve some arbitrariness.

Let us define the wavenumber $k_{0}$ as the wavenumber of the largest eddy in the inertial range. Thus we relate $k_{0}$ to the mixing length as follows:

$k_{0}=\frac{2 \pi}{\beta \Lambda}$

where the parameter $\beta$ is introduced here again in order to gauge the definition of $k_{0}$.

For convenience we define the following variables:

$K=\frac{k}{k_{0}}, u_{K}=\frac{u_{k}}{u_{0}}, \omega_{\Lambda}=\frac{2 \pi u_{0}}{\Lambda}$.

The mean square amplitude of Eq. (58) for the Gaussian form of Eq. (30) is given by

$$
\begin{aligned}
\left\langle|A|^{2}\right\rangle= & \frac{\lambda \beta^{4}}{32 \pi \eta\left(\omega_{0} I\right)^{2}} \int_{0}^{M} \mathrm{~d} m \rho_{0} u_{0}^{3} \Lambda^{4}\left(\frac{\mathrm{d} \xi_{r}}{\mathrm{~d} r}\right)^{2} \\
& \times\left[\mathcal{G} \mathcal{S}_{\mathrm{R}}(X)+\mathcal{H} \mathcal{R}^{2} \mathcal{F}^{2} \mathcal{S}_{\mathrm{S}}(X)\right]
\end{aligned}
$$

with the "source functions"

$$
\begin{aligned}
\mathcal{S}_{\mathrm{R}}(X)= & \frac{1}{2 \sqrt{\pi}} \int_{0}^{\infty} \frac{\mathrm{d} K}{K^{3}} \frac{1}{u_{K}} \frac{k_{0}^{2} E^{2}(K)}{u_{0}^{4}} \mathrm{e}^{-Z^{2}} \\
\mathcal{S}_{\mathrm{S}}(X)= & \frac{X^{-2}}{2(2 \pi)^{5 / 2}} \int_{0}^{\infty} \frac{\mathrm{d} K}{K^{3}} \frac{1}{u_{K}} \\
& \times\left(\frac{k_{0} E_{\mathrm{S}}(K)}{\tilde{s}^{2}}\right)\left(\frac{k_{0} E(K)}{u_{0}^{2}}\right) \mathrm{e}^{-(Z \sqrt{2})^{2}},
\end{aligned}
$$

where $X\left(m, \omega_{0}\right) \equiv \omega_{0} / \omega_{\Lambda}$ and the ratio $Z=\lambda \beta X / 2 K u_{K}$. As shown below, the ratios $k_{0} E_{S}(K) / \tilde{s}^{2}$ and $k_{0} E(K) / u_{0}^{2}$ only depend on $K$.

The amplitude (Eq. (65)) is very sensitive to the parameters $\beta$ and $\lambda$ because it scales as $\lambda \beta^{4}$ and the quantity $\lambda \beta$ is involved in the exponential function of Eq. (66) and Eq. (67). In Sect. 5.2 some physical arguments yield a crude estimate for the parameter $\beta$. Values of parameters $\lambda, \beta$ are discussed in some details in Samadi et al. (2001a) in connection with solar seismic observations.

\subsection{Kolmogorov energy spectrum}

The normalization condition Eq. (52) allows us to express the Kolmogorov spectrum (Lesieur 1997, Chap. VI-4) as

$E(K)=\frac{u_{0}^{2}}{k_{0}} K^{-5 / 3}$ for $K>1$.

The expression for the mean square amplitude (Eq. (58)) involves also the entropy spectrum $E_{\mathrm{s}}(k)$ which must therefore be determined. We recall that the energy spectra of the temperature and of the entropy fluctuations 
exhibit the same behavior (see Sect. 4.1). Turbulence theory predicts that the energy spectrum of the temperature fluctuations follows the same scaling law as that of the kinetic energy spectrum $E(k)$ in the inertial-convective range (Lesieur 1997, Chap. VI-10) and it decreases as $k^{-17 / 3}$ in the inertial-conductive range because molecularconductive effects are predominant. This behavior seems to be quite well supported by observations of solar granulation as is claimed by Espagnet et al. (1993) and Nesis et al. (1993).

We next turn to a specific case where we assume that the entropy energy spectrum lies in the inertial-convective range i.e. the turbulent entropy spectrum scales as the kinetic spectrum. According to the normalization conditions of Eq. (52) and of Eq. (55), this assumption provides the relation:

$\frac{E_{\mathrm{s}}(k, \omega)}{\tilde{s}^{2}}=\frac{1}{3} \frac{E(k, \omega)}{u_{0}^{2}}$.

The source functions with Eq. (68) and Eq. (69), Eq. (66) and Eq. (67) become:

$\mathcal{S}_{\mathrm{R}}(X) \simeq 0.92 \int_{1}^{\infty} \mathrm{d} K \frac{u_{K}^{3}}{K^{5}} \mathrm{e}^{-Z^{2}}$
$\mathcal{S}_{\mathrm{S}}(X) \simeq \frac{0.008}{X^{2}} \int_{1}^{\infty} \mathrm{d} K \frac{u_{K}^{3}}{K^{5}} \mathrm{e}^{-(Z / \sqrt{2})^{2}}$

with $X$ and $Z$ defined below Eq. (67). The source function $S_{\mathrm{R}}(X)$ (Eq. 70) is similar to the source function $S_{\mathrm{Q}}(m, \omega)$ obtained by B92 (Eq. (2.20)). However the source function for the entropy contribution $S_{\mathrm{S}}(X)$ (Eq. (71)) differs from the one established by B92 (Eq. (3.9)) in that the author extrapolated GK's formulation of the Reynolds stress contribution for the contribution due to the Lagrangian entropy fluctuations.

For sake of comparison with the GMK formulation, we simplify Eqs. (70) and (71) by using the fact that most of the stochastic emission occurs from eddies with $\omega_{k} \gtrsim \omega_{0}$ (i.e. with $\left.\mathrm{e}^{-\left(\omega_{0} / \omega_{k}\right)^{2}} \sim 1\right)$. For $\omega_{0} / \omega_{\Lambda} \gtrsim 1$ integration over $K$ leads then to the asymptotic forms

$\mathcal{S}_{\mathrm{R}} \propto\left(\omega_{0} / \omega_{\Lambda}\right)^{-15 / 2}$ for $\frac{\omega_{0}}{\omega_{\Lambda}} \gtrsim 1$

$\mathcal{S}_{\mathrm{S}} \propto\left(\omega_{0} / \omega_{\Lambda}\right)^{-19 / 2}$ for $\frac{\omega_{0}}{\omega_{\Lambda}} \gtrsim 1$

The asymptotic frequency dependence, Eq. (72), is as that found by GMK. On the other hand, GMK assume the same frequency dependence for the two contributions while here the source function $\mathcal{S}_{\mathrm{S}}(\mathrm{Eq}$. (73)) corresponding to the entropy contribution exhibits a steeper slope. This result shows us that the power emission from the entropy contribution is less efficient at high frequency compared to the Reynolds term and thus differs from the results of GMK but is consistent with the GK statement that the contribution of the entropy fluctuation is larger for the long period $p$-modes than for the short period $p$-modes.

\subsection{Constraints on free parameters}

We next turn to the parameter $\beta$. The time scale at which the convective energy dissipates through the turbulent cascade is of order $\Lambda / w$. Thus for stationary turbulence and using Eq. (52), the rate of injection of kinetic energy $\epsilon$ (Lesieur 1997, Chap. VI-3) can be crudely estimated as

$\epsilon \simeq \frac{3}{2} u_{0}^{2} w / \Lambda$

As $E(k)=C_{K} \epsilon^{2 / 3} k^{-5 / 3}$ (see Lesieur 1997, Chap. VI-4) where $C_{\mathrm{K}}$ is the Kolmogorov universal constant, which is close to 1.5, and from Eqs. (68) and (74) one obtains

$k_{0} \simeq \frac{3}{2} C_{\mathrm{K}}^{3 / 2}(\Phi / 3)^{-1 / 2} \Lambda^{-1}$

and then

$\beta \simeq \frac{4 \pi}{3} C_{\mathrm{K}}^{-3 / 2}(\Phi / 3)^{1 / 2}$.

This suggests that crudely $\beta \simeq 1.9$ with $\Phi=2$, the value of the anisotropic factor consistent with BV's MLT.

The value suggested for $\beta$ is somewhat approximate. Therefore, as in the case of the parameter $\lambda$, we consider $\beta$ as a free parameter. However, the value of $\beta \lambda$ is constrained by an upper limit. Indeed, let $\tau_{k_{0}}$ be the correlation time of the largest eddy in the inertial domain. The lifetime of the largest eddies in the inertial range cannot be longer than the characteristic time $\Lambda / w$ at which the convective energy dissipates into the turbulent cascade. Therefore we must have $\tau_{k_{0}} \lesssim \Lambda / w$ and according to Eqs. $(61,62)$ evaluated for $k=k_{0}$ and Eqs. $(63,52)$ we obtain $\beta \lambda \lesssim 2.7 \Phi^{1 / 2}$.

\section{Discussion}

In the present work, a formulation has been established which yields the oscillation amplitude of a stellar oscillation mode when it is stochastically excited by turbulent convection. The main result of this paper is the expression for the mean square oscillation amplitude $\left\langle|A|^{2}\right\rangle$ given by Eqs. (58) and (59). The derivation is based on theoretical developments of previous works (GK, GMK, B92) but an effort has been made to obtain a sufficiently general expression which enables one to implement any type of turbulent (kinetic and entropic) spectra and any type of temporal spectra for the turbulent eddies.

For comparison purpose, we next focused on a Gaussian temporal and Kolmogorov energy spectra; we then ended up with the same expression for the Reynolds stress contribution as obtained in GMK and B92. We must stress however that in order to use the same formulation for an energy spectrum other than the Kolmogorov one, such as for instance the Spiegel spectrum, a general expression such as Eq. (58) must be used.

As far as the entropy contribution is concerned, we found that the linear term due to the entropy fluctuation gives no contribution and that it is the advection of 
the Eulerian entropy fluctuations by the turbulent velocity field which produces a nonzero acoustic emission.

In the derivation of the expected mean-square amplitude (Eq. (58)), several assumptions and approximations have been made. For instance, it has been assumed that the oscillations and the stratification are decoupled for the eddies which contribute to the stochastic power emission. This assumption was shown to be valid and is in agreement with Goldreich \& Kumar (1990). In addition, we have used the plane-parallel approximation, which is valid in the excitation region. Other assumptions are based on results from Stein's work (1967), such as the separation of the kinetic energy spectrum $E(k, \omega)$ in term of a purely spatial energy spectrum $E(k)$ and a time-dependent factor $\chi_{k}(\omega)$ for an eddy of wavenumber $k$. As in Stein (1967) the QNA has been used. We have used crude approximations for estimations of the velocity and the life-time of an eddy as proposed by Stein (1967). This led us to introduce the free parameter $\lambda$ in the definition of the eddy lifetime.

The entropy fluctuation has been considered to act as a passive scalar and we have extended the separation of the kinetic energy spectrum in terms of a purely spatial energy spectrum $E(k)$ and a time-dependent factor to the entropy energy spectrum.

The MLT was required in order to estimate the power injected in the velocity and entropy turbulent cascade (Eq. (65)). This theory, which assumes the Boussinesq approximation, is well known to be a crude approximation. For instance the MLT predicts that the characteristic size of the largest turbulent element is comparable with the scale height of the stratification. This is in contrast with the homogeneous hypothesis considered here for the description of the turbulent medium. However, it has already been stressed that the stochastic emission is not affected by the stratification. The use of an homogeneous turbulence is therefore valid.

The size of the largest eddy in the inertial range is estimated by the mixing length $(\Lambda)$ according to the MLT. We have related the wavenumber of the largest eddy in the inertial range $\left(k_{0}\right)$ to the mixing length. However, as for the eddy lifetime, this relation is rather arbitrary and therefore involves uncertainties. We have therefore introduced an additional free parameter $\beta$.

We have considered an isotropic turbulence. However, effects of anisotropy in the amplitude computaion have been partially taken into account. In this way, two anisotropic factors $\mathcal{H}$ and $\mathcal{G}$ have been introduced for both contributions respectively. These factors have been related to Gough (1977) anisotropic factor $\Phi$.

It is possible to validate some of these approximations by comparing them with results of $3 \mathrm{D}$ simulations of the solar envelope (Samadi 2000, work in progress): for instance, the QNA is found to be reasonably valid.

To date, and in the solar case, several possible turbulent spectra can be investigated and compared with solar seismic observations (Samadi et al. 2001a). But we can anticipate that the entropy contribution will be dominant, as already pointed out by GMK. Comparison with solar data allows us to calibrate the free parameters which in turn can be used to compute oscillation power spectra for various solar-like oscillation stars. Indeed, unlike the Sun, it is not possible to determine the turbulent spectra of other stars from observations of the surface granulation. In the prospect of forthcoming space seismic projects (COROT, MONS, MOST, EDDINGTON) comparison of theoretical computations with seismic data of several solar-like oscillation stars will provide useful constraints on stellar turbulent spectra.

Acknowledgements. We are indebted to A. Mangeney for particularly useful suggestions and advice without which this work would not have been possible. We also thank J.-P. Zahn, M. Rieutord and A. Baglin for useful discussions.

\section{Appendix A: Crossing terms}

The entropy and Reynolds source terms involve three crossing terms arising from Eq. (21). These terms are proportional to

$$
\begin{array}{r}
\int \mathrm{d}^{3} r\left\langle s^{\prime} u_{i}^{\prime \prime} u_{j}^{\prime \prime}\right\rangle \\
\int \mathrm{d}^{3} r\left\langle u_{l}^{\prime} s^{\prime} u_{i}^{\prime \prime} u_{j}^{\prime \prime}\right\rangle
\end{array}
$$

The QNA gives the following relation

$$
\begin{aligned}
\left\langle u_{l}^{\prime} s^{\prime} u_{i}^{\prime \prime} u_{j}^{\prime \prime}\right\rangle=\left\langle u_{l}^{\prime} s^{\prime}\right\rangle\left\langle u_{i}^{\prime \prime} u_{j}^{\prime \prime}\right\rangle & +\left\langle u_{l}^{\prime} u_{i}^{\prime \prime}\right\rangle\left\langle s^{\prime} u_{j}^{\prime \prime}\right\rangle \\
& +\left\langle u_{l}^{\prime} u_{j}^{\prime \prime}\right\rangle\left\langle s^{\prime} u_{i}^{\prime \prime}\right\rangle .
\end{aligned}
$$

For an isotropic, homogeneous and incompressible turbulence we have $\left\langle s^{\prime} u_{j}^{\prime \prime}\right\rangle=0$ (Lesieur 1997, Chap. V-8). Thus the three terms in the RHS of Eq. (A.3) vanish.

The entropy source term introduces a crossing term between the linear and the non-linear terms of Eq. (39). This term is proportional to

$\int \mathrm{d}^{3} r\left\langle s^{\prime} s^{\prime \prime} u_{i}^{\prime \prime}\right\rangle$

Third order correlation products of quantities following a normal distribution are expected to be equal to zero. Turbulent quantities, such as the entropy fluctuations $\left(s_{\mathrm{t}}\right)$ and the turbulent velocity field $(\boldsymbol{u})$, are not Gaussian random functions, since such turbulence would have no energy transfer between wavenumbers (Lesieur 1997). However, for a strongly turbulent medium, as in a stellar convection zone, approximation of turbulent quantities by Gaussian random functions is consistent with the QNA and can therefore be considered as valid.

As a conclusion, the crossing term $C_{\mathrm{RS}}$ and the crossing terms between the linear and non-linear terms of the entropy contribution do not contribute to oscillation excitation. 


\section{Appendix B: Entropy contribution}

The QNA provides the relation

$$
\begin{aligned}
\left\langle u_{3}^{i} u_{4}^{j} s_{3} s_{4}\right\rangle=\left\langle u_{3}^{i} u_{4}^{j}\right\rangle\left\langle s_{3} s_{4}\right\rangle & +\left\langle s_{3} u_{3}^{i}\right\rangle\left\langle s_{4} u_{4}^{j}\right\rangle \\
& +\left\langle s_{3} u_{4}^{j}\right\rangle\left\langle s_{4} u_{3}^{i}\right\rangle .
\end{aligned}
$$

The terms of type $\left\langle u_{3}^{i} s_{3}\right\rangle$ and $\left\langle u_{3}^{i} s_{4}\right\rangle$ vanish (Lesieur 1997, Chap. V-8) and Eq. (48) reduces to

$$
\begin{aligned}
C_{\mathrm{S}}^{2}= & \int \mathrm{d}^{3} x_{0}\left(\alpha_{s} \boldsymbol{\nabla}_{0} \cdot \boldsymbol{\xi}\right)^{2} g_{i j} \int \mathrm{d} \tau \mathrm{e}^{-i \omega_{0} \tau} \int \mathrm{d}^{3} r \\
& \times \int_{-\infty}^{\tau / 2} \mathrm{~d} t_{3} \int_{-\infty}^{-\tau / 2} \mathrm{~d} t_{4}\left\langle u_{3}^{i} u_{4}^{j}\right\rangle\left\langle s_{3} s_{4}\right\rangle .
\end{aligned}
$$

Stationarity of the turbulence allows us to write Eq. (B.2) as

$$
\begin{aligned}
C_{\mathrm{S}}^{2} \propto & \int \mathrm{d} \tau \mathrm{e}^{-i \omega_{0} \tau} \mathrm{d}^{3} r \int_{-\infty}^{\tau / 2} \mathrm{~d} t_{3} \\
& \times \int_{-\infty}^{-\tau / 2} \mathrm{~d} t_{4}\left\langle u^{i} u^{j}\left(t_{4}-t_{3}\right)\right\rangle\left\langle s_{\mathrm{t}} s_{\mathrm{t}}\left(t_{4}-t_{3}\right)\right\rangle
\end{aligned}
$$

which can be expanded in the Fourier space as

$$
\begin{aligned}
C_{\mathrm{S}}^{2} \propto & (2 \pi)^{3} \int \mathrm{d} \tau \mathrm{e}^{-i \omega_{0} \tau} \mathrm{d}^{3} k \\
& \times \int_{-\infty}^{\tau / 2} \mathrm{~d} t_{3} \int_{-\infty}^{-\tau / 2} \mathrm{~d} t_{4} \int \mathrm{d} \omega_{3} \mathrm{~d} \omega_{4} \\
& \times \phi_{i j}\left(\boldsymbol{k}, \omega_{3}\right) \phi_{s}\left(\boldsymbol{k}, \omega_{4}\right) \mathrm{e}^{-i t_{3}\left(\omega_{3}+\omega_{4}\right)} \mathrm{e}^{i t_{4}\left(\omega_{3}+\omega_{4}\right)} .
\end{aligned}
$$

Integrating over $t_{3}$ and $t_{4}$ in Eq. (B.4) and then over $\tau$ yields:

$C_{\mathrm{S}}^{2} \propto \frac{(2 \pi)^{4}}{\omega_{0}^{2}} \int \mathrm{d}^{3} k \int \mathrm{d} \omega \phi_{i j}(\boldsymbol{k}, \omega) \phi_{s}\left(\boldsymbol{k}, \omega_{0}+\omega\right)$.

According to Eqs. $(28,29,42,46)$, Eq. (B.5) can be written as

$$
\begin{gathered}
C_{\mathrm{S}}^{2}=\frac{(2 \pi)^{4}}{\omega_{0}^{2}} \int \mathrm{d}^{3} x_{0}\left(\alpha_{s} \boldsymbol{\nabla}_{0} \cdot \boldsymbol{\xi}\right)^{2} g_{i j} \int \mathrm{d} \omega \int \mathrm{d}^{3} k \\
\times \frac{E(k)}{4 \pi k^{2}}\left(\delta_{i j}-\frac{k_{i} k_{j}}{k^{2}}\right) \frac{E_{\mathrm{S}}(k)}{2 \pi k^{2}} \chi_{k}\left(\omega_{0}+\omega\right) \chi_{k}(\omega) .
\end{gathered}
$$

\section{Appendix C: Anisotropy effects}

We establish here the derivation of Eqs. (34) and (50) where the anisotropy factors $\mathcal{G}$ and $\mathcal{H}$ have been introduced. Thus we evaluate these factors for several cases. Let $(x, y, z)$ be the real (anisotropic) coordinates and $(\tilde{x}, \tilde{y}, \tilde{z})$ are isotropic coordinates. We assume an isotropic turbulence in the horizontal layers while the anisotropy occurs in the vertical direction only. The two sets of coordinates are then related to each other by the anisotropic factor $\mathcal{Q}$ as

$x=\mathcal{Q} \tilde{x} \quad y=\mathcal{Q} \tilde{y} \quad z=\tilde{z}$.

$\mathcal{Q}$ is also the ratio of the horizontal to vertical correlation lengths of the eddies. The equivalent relation in the wavenumber space is

$k_{x}=\mathcal{Q}^{-1} \tilde{k}_{x} \quad k_{y}=\mathcal{Q}^{-1} \tilde{k}_{y} \quad k_{z}=\tilde{k}_{z}$ where $\left(k_{x}, k_{y}, k_{z}\right)$ and $\left(\tilde{k}_{x}, \tilde{k}_{y}, \tilde{k}_{z}\right)$ are anisotropic and isotropic wavenumber coordinates respectively. $\mathcal{Q}=1$ corresponds to an isotropic turbulence. We place ourselves in the case $\mathcal{Q} \neq 1$ but suppose that the energy spectra remain unchanged i.e. consistent with an isotropic turbulence. We therefore model only geometrical effects due to non-isotropic shapes of the eddies.

Evaluation of Eq. (33) gives

$$
\begin{aligned}
C_{\mathrm{R}}^{2}= & 4 \pi^{3} \int_{0}^{M} \mathrm{~d} m \rho_{0}\left(\frac{\mathrm{d} \xi_{r}}{\mathrm{~d} r}\right)^{2} \\
& \times \int \mathrm{d} k \frac{E^{2}(k)}{k^{2}} \chi_{k}\left(\omega_{0}\right) \\
& \times \int \mathrm{d} \theta \sin \theta\left(1-\frac{\mathcal{Q}^{2} \cos ^{2} \theta}{\left(\mathcal{Q}^{2}-1\right) \cos ^{2} \theta+1}\right)^{2} .
\end{aligned}
$$

We define for convenience the factor $\mathcal{G}$ as

$$
\mathcal{G}=\int_{-1}^{1} \mathrm{~d} \cos \theta\left(1-\frac{\mathcal{Q}^{2} \cos ^{2} \theta}{\left(\mathcal{Q}^{2}-1\right) \cos ^{2} \theta+1}\right)^{2}
$$

This leads to Eq. (34).

As was done with the Reynolds stress contribution, it is straightforward to obtain Eq. (50) from Eq. (B.6) where the anisotropic factor $\mathcal{H}$ is

$\mathcal{H}=\int_{-1}^{1} \mathrm{~d} \cos \theta\left(1-\frac{\mathcal{Q}^{2} \cos ^{2} \theta}{\left(\mathcal{Q}^{2}-1\right) \cos ^{2} \theta+1}\right)$.

Gough (1977) defines the velocity anisotropic factor $\Phi$ as

$$
\Phi=\frac{\left\langle u^{2}\right\rangle}{\left\langle u_{z}^{2}\right\rangle}
$$

The lifetime of an eddy is the same in any direction we then have

$$
\frac{\mathcal{Q} \Lambda}{\left\langle u_{x}^{2}\right\rangle^{1 / 2}}=\frac{\mathcal{Q} \Lambda}{\left\langle u_{y}^{2}\right\rangle^{1 / 2}}=\frac{\Lambda}{\left\langle u_{z}^{2}\right\rangle^{1 / 2}}
$$

where $\Lambda$ is the correlation length of the largest eddy in the vertical direction. Thus one obtains from Eqs. (C.6, C.7) a relation between $\mathcal{Q}$ and $\Phi$

$\Phi=1+2 \mathcal{Q}^{2}$.

Integration of Eqs. (C.4 , C.5) gives

$$
\begin{aligned}
& \mathcal{G}=\frac{1}{a^{5 / 2}}\left[\frac{2 a^{3 / 2}-3 \sqrt{a}}{a-1}-3 \operatorname{atanh}(\sqrt{a})\right] \\
& \mathcal{H}=\frac{2}{a^{3 / 2}}[\operatorname{atanh}(\sqrt{a})-\sqrt{a}]
\end{aligned}
$$

with

$a=1-\mathcal{Q}^{2}=(3-\Phi) / 2$.

Table C.1 gives values for $\mathcal{G}$ and $\mathcal{H}$ for different values of $\Phi$. 
Table C.1. Values for $\mathcal{H}$ and $\mathcal{G}$ for several cases: the isotropic case corresponds to $\Phi=3 ; \Phi=2$ is consistent with BohmVitense's MLT; $\Phi=5 / 3$ is the value that maximizes the convective heat fluxes (Balmforth \& Gough 1990) and $\Phi=1.37$ is the value considered by Houdek et al. (2001)

\begin{tabular}{lllll}
\hline$\Phi$ & $\mathcal{Q}^{2}$ & $a$ & $\mathcal{G}$ & $\mathcal{H}$ \\
\hline 3 & 1 & 0 & $16 / 15$ & $4 / 3$ \\
2 & 0.5 & 0.5 & 1.0 & 1.0 \\
$5 / 3$ & 0.33 & 0.66 & 1.8 & 1.2 \\
1.37 & 0.18 & 0.81 & 3.7 & 1.6 \\
\hline
\end{tabular}

\section{References}

Baglin, A., \& The Corot Team. 1998, in IAU Symp. 185: New Eyes to See Inside the Sun and Stars, vol. 185, 301

Balmforth, N. J. 1992, MNRAS, 255, 639

Balmforth, N. J., \& Gough, D. O. 1990, Sol. Phys., 128, 161

Batchelor, G. K. 1970, The theory of homogeneous turbulence (University Press)

Böhm-Vitense, E. 1958, Z. Astrophys., 46, 108

Christensen-Dalsgaard, J., \& Frandsen, S. 1983, Sol. Phys., 82, 165

Cowling, T. G. 1941, MNRAS, 101, 367

Cox, J. 1968, Principles of stellar structure (Gordon and Breach)

Espagnet, O., Muller, R., Roudier, T., \& Mein, N. 1993, A\&A, 271,589

Favata, F., Roxburgh, I., \& Christensen-Dalsgaard, J. 2000, in The Third MONS Workshop: Science Preparation and Target Selection, 49

Goldreich, P., \& Keeley, D. A. 1977, ApJ, 212, 243

Goldreich, P., \& Kumar, P. 1990, ApJ, 363, 694

Goldreich, P., Murray, N., \& Kumar, P. 1994, ApJ, 424, 466

Gough, D. O. 1977, ApJ, 214, 196

Houdek, G., Balmforth, N. J., Christensen-Dalsgaard, J., \& Gough, D. O. 1999, A\&A, 351, 582
Houdek, G., Chaplin, W., Appourchaux, T., et al. 2001, submitted to MNRAS

Kjeldsen, H., \& Bedding, T., eds. 1998, The First MONS Workshop: Science with a Small Space Telescope, ed. H. Kjeldsen, \& T. Bedding, Aarhus Universitet

Kraichnan, R. H. 1957, Phys. Rev., 107, 1485

Lesieur, M. 1997, Turbulence in fluids (Kluwer Academic Publishers)

Lighthill, M. J. 1952, Proc. R. Soc. Lond., A211, 564

Matthews, J. M. 1998, in Structure and Dynamics of the Interior of the Sun and Sun-like Stars, 395

Musielak, Z. E., Rosner, R., Stein, R. F., \& Ulmschneider, P. 1994, ApJ, 423, 474

Nesis, A., Hanslmeier, A., Hammer, R., Komm, R., Mattig, W., \& Staiger, J. 1993, A\&A, 279, 599

Osaki, Y. 1990, in Lecture Notes in Physics: Progress of Seismology of the Sun and Stars, ed. Y. Osaki, \& H. Shibahashi (Springer-Verlag), 75

Samadi, R. 2000, Ph.D. Thesis, Université Paris 6

Samadi, R., Goupil, M.-J., \& Lebreton, Y. 2001a, A\&A, 370, 147

Samadi, R., Goupil, M.-J., Lebreton, Y., \& Baglin, A. 2001b, in Proceedings of the SOHO 10/GONG 2000 Workshop, Helioand Asteroseismology at the Dawn of the Millennium, ed. A. Wilson, vol. SP-464 (ESA Publications Division), 451 [astro-ph/0101129]

Samadi, R., Houdek, G., Goupil, M.-J., \& Lebreton, Y. 2001c, submitted to A\&A

Stein, F. \& Nordlund, A. 1991, in Challenges to Theories of the Structure of Moderate-Mass Stars, vol. 195, ed. D. Gough, \& J. Toomre (Springer-Verlag)

Stein, R. F. 1967, Solar Phys., 2, 385

Tennekes, H. 1975, J. Fluids Mech., 67, 561

Tennekes, H., \& Lumley, J. 1982, A first course in turbulence, 8th ed. (MIT Press)

Unno, W., Osaki, Y., Ando, H., Saio, H., \& Shibahashi, H. 1989, Nonradial oscillations of stars (Tokyo: University of Tokyo Press, 1989, 2nd ed.) 